\title{
La enseñanza del cuidado de enfermería en una Facultad de Enfermería en Bogotá1.
}

\author{
Lina María Vargas-Escobar ${ }^{2 *}$, Ana Cecilia Becerra Pabón ${ }^{3}$ \\ ${ }^{1}$ En el marco del proyecto titulado: Lineamientos curriculares para la enseñanza del cuidado de enfermería en el programa de Enfermería de una Institución de \\ Educación Superior de la ciudad de Bogotá. Financiado por convocatoria interna PCI 2015- 8158, Universidad El Bosque. Bogotá (Colombia). \\ ${ }^{2}$ Enfermera, Magíster y Doctora en Enfermería. Profesora Asociada. Facultad de Enfermería, Universidad El Bosque. Bogotá (Colombia). \\ ${ }^{3}$ Enfermera, Doctora en Ciencias de la Educación, profesora Universidad El Bosque hasta diciembre de 2016. En la actualidad: Coordinadora de Investigación - \\ Escuela de Enfermería, Fundación Universitaria Juan N. Corpas - Escuela de Enfermería. Bogotá (Colombia).
}

\section{RESUMEN}

Introducción. La enseñanza del cuidado de Enfermería, es la piedra angular sobre la que se sostiene la disciplina y los procesos de profesionalización. Es imperativo generar investigaciones que profundicen la forma en que la universidad está abordando este concepto y de qué manera se desarrolla desde el documento hasta el profesional.

Objetivos. Comprender aspectos curriculares y vivenciales de estudiantes y profesores acerca de la formación en el cuidado de enfermería enmarcado en el modelo biopsicosocial, cultural y espiritual abordado por un programa de enfermería en Bogotá.

Método. Paradigma cualitativo y de análisis de contenido, que se llevó a cabo en dos fases. Fase 1: revisión documental de los syllabus y proyecto educativo del programa de Enfermería (PEP). Fase 2: entrevistas a profundidad a estudiantes de último semestre y a profesores del programa de enfermería durante el periodo 2017 y 2018.

Resultados. Fase 1: El cuidado de enfermería de los documentos del programa, se enmarcan en las siguientes categorías temáticas: definición de un cuidado desde la reciprocidad, abordaje de situaciones de salud enfermedad, gerencia y gestión del cuidado, enfoque biopsicosocial, cultural y espiritual, cuidado integral, habilidades y competencias para el cuidado.

Fase 2: Para los estudiantes el cuidado se enmarca en tres categorías: cuidado con enfoque biopsicosocial durante los procesos de salud-enfermedad, cuidado holístico y conocimientos teóricoprácticos del cuidado. Para los docentes el cuidado está inmerso en tres categorías: cuidado desde una concepción individua Vs cuidado de enfermería, un cuidado que privilegia el componente físico y propuesta para la enseñanza y la formación en el cuidado

Conclusiones. En el programa de enfermería se ofrece un cuidado con enfoque biopsicosocial, cultural y espiritual, vinculado en su mayoría a un paradigma de reciprocidad, que debe seguirse fortaleciendo desde el dialogo entre docentes, estrategias pedagógicas y una comprensión más integral y total del cuidado de enfermería.

Palabras clave: Educación en enfermería; Facultades de Enfermería; Docentes de Enfermería; Estudiantes de Enfermería (DeCS).

\section{ABSTRACT}

\section{Teaching Nursing Care in a School of Nursing in Bogotá.}

Introduction. The education and training of nursing professionals in nursing care is the cornerstone of the discipline and professionalization processes.

Objectives. To understand curricular and experiential aspects of students and teachers regarding nursing care training within the framework of a biopsychosocial, cultural and spiritual model approached by a Nursing Program in Bogotá.

Method. Qualitative paradigm and content analysis, which was carried out in two phases. Phase 1: Documentary review of the syllabi and the educational project of the nursing program (EPP). Phase 2: Indepth interviews with nursing students in their last semester and nursing teachers during the period 2017 to 2018 .

Results. Phase 1. Nursing care according the curriculum documents is framed by the following thematic categories: definition of care based on reciprocity view; approach to health-disease processes; care management; biopsychosocial, cultural, and spiritual approach; integral care; care skills and competencies. Phase 2. For students, nursing care is framed by three categories: biopsychosocial approach to care during health-disease processes, holistic care, and theoreticalpractical knowledge of care. For teachers, the care is framed by three categories: care from a personal point of view vs. nursing care, nursing care that favors the physical component, and a proposal for care teaching and training in care.

Conclusions. The nursing program offers training in nursing care under a biopsychosocial, cultural, and spiritual focus, linked to the reciprocal interaction paradigm, which should continue to be strengthened through dialogue among teachers, pedagogical strategies, and a more comprehensive and whole understanding of nursing care.

Keywords: Nursing Education; Nursing Faculties; Nursing Teachers; Nursing Students (DeCS)

Como citar: Vargas-Escobar, LM, Becerra Pabón, AC. La enseñanza del cuidado de enfermería en una Facultad de Enfermería en Bogotál. Revista Cuarzo 2019;25(2):8-20.

Recibido: 10 de julio de 2019 Aceptado: 18 de septiembre de 2019 Publicado: 30 de diciembre de 2019

DOI: https://doi.org/10.26752/cuarzo.v25.n2.424 


\section{INTRODUCCIÓN}

$\mathrm{L}$ a enfermería es una disciplina profesional que desde hace varios años viene desarrollando un proceso de análisis, reflexión y construcción de conocimientos alrededor de aquello que da sustento a su práctica y a su razón de ser; esto es el cuidado de enfermería. Diversas teóricas como como Peplau, Orlando, King, Levine, Roy, Leininger, Newman, Watson, Rogers y Parse han establecido el cuidado como la esencia o el dominio central de la enfermería (1).

En Colombia, la Ley 911 de 2004 establece el "Acto de cuidado", como el ser y la esencia de la profesión. En ella, el artículo 3 establece que: "Se fundamenta en sus propias teorías y tecnologías y en conocimientos actualizados de las ciencias biológicas, sociales y humanísticas. El artículo explica que el cuidado se da a partir de la comunicación y relación interpersonal humanizada entre el profesional de enfermería y el ser humano, sujeto de cuidado, la familia o grupo social, en las distintas etapas de la vida, situación de salud y del entorno". Establece además que a través del plan de cuidado de enfermería se ofrece este cuidado con el propósito de promover la vida, prevenir la enfermedad, intervenir en el tratamiento, en la rehabilitación y ofrecer cuidado paliativo con el fin de desarrollar, en la medida de lo posible las potencialidades de individuales y colectivas (2).

En este sentido, el concepto del cuidado se convierte en objeto de reflexión propia para la disciplina y sus practicantes, lo cual permite no solo hacer una diferenciación definitiva de los paradigmas biomédicos o de otras ciencias de la salud, sino que a su vez plantea los nuevos roles que tiene el profesional de enfermería en la sociedad, frente al contexto, las necesidades propias de salud de la población colombiana, la nueva evidencia disponible y sus aportes a la misma. Por esta razón, el cuidado de enfermería hace parte de la formación de los futuros profesionales de enfermería y es introducido en los estudiantes como un eje fundamental de los currículos de enfermería en la formación disciplinar (3).

La discusión y reflexión frente a los roles del profesional de enfermería y su ejercicio de cuidado en la práctica debe estar presente en las discusiones de los Comités de Currículo de las Facultades de Enfermería, los procesos de registro calificado y acreditación de los programas de enfermería en Colombia.

En la literatura se encuentran estudios como los de Oliveira Salvador y colaboradores que exploran la enseñanza del cuidado de enfermería en el ámbito técnico desde una perspectiva de los docentes (4), Rodríguez J y colaboradores que revisaron la enseñanza del cuidado de enfermería en la salud mental (5), Rojas Espinosa y colaboradores que estudiaron la relación del contenido de los currículo de tres instituciones educativas formadoras de licenciadas (os) en enfermería y la percepción que los alumnos tienen sobre el cuidado profesional (6), entre otros; que dan cuenta de la forma en se ve el cuidado de enfermería en sus programas de enfermería. Otros que reflexionan sobre lo que es la enfermería en la actualidad y cómo debe enseñarse como el de Wikström BM (7) o Gómez y colaboradores (8), entre otros; que dan cuenta del interés que existe en la actualidad y que seguirá en el futuro por el estudio de esta área temática. Sin embargo, existen pocos estudios que den cuenta de cómo influye la formación académica que reciben los estudiantes de enfermería, en su concepción o sentido del cuidado, después de culminado su formación profesional universitaria; así como los aspectos significativos aportan los profesores en la formación de esta concepción del cuidado. Por esta razón, en el presente estudio se pretende responder a la siguiente pregunta de investigación ¿cómo es experimentado el currículo por estudiantes y docentes frente a la formación del cuidado de enfermería enmarcado en el enfoque biopsicosocial, cultural y espiritual en una IES?

El poder dar respuesta a este planteamiento, ofrecerá información para comprender aspectos metodológicos, conceptuales y curriculares que influyen en la formación del cuidado en un programa de enfermería, con el fin de seguir fortaleciendo el proceso formativo alrededor del objeto central de enfermería en los futuros enfermeros de un programa de enfermería de la ciudad de Bogotá, Colombia.

\section{MATERIALES Y MÉTODOS}

El presente estudio se asume bajo el paradigma cualitativo de la investigación y de análisis de contenido, el cual se llevó a cabo en dos fases: a) Revisión documental de los syllabus del programa y proyecto educativo del programa de Enfermería (PEP). b) Entrevistas a profundidad a estudiantes de último semestre y a profesores del programa de enfermería, hasta que se logró la saturación de la información.

Para el análisis de la información de la primera fase, se construyó una matriz de registro de información para el PEP (Proyecto Educativo del Programa) que contenía los siguientes elementos: Misión, Visión, Plan de estudio, Perfiles, Justificación, Contenidos, Objetivos (Objetivo de conocimiento, objetivos de integración, Objetivos de compromiso, Objetivos de dimensión humana), Actividades y Evaluación. También se construyó otra por las tres áreas: profesional, básica y socio-humanística, que incluyó el nombre de la asignatura, el componente del syllabus a estudiar, el registro explícito o implícito del cuidado y comentarios.

Para el análisis de la segunda fase, las entrevistas realizadas fueron trascritas y analizadas posteriormente con el Software Atlas ti 7.0, por medio de un proceso de análisis de contenido.

De acuerdo a la Resolución 8430 de 1993 (9) esta investigación se considera como "Investigación sin riesgos". Para salvaguardar los aspectos éticos del estudio, el estudio obtuvo el aval por el Comité Ético de la Universidad El Bosque y se administró un consentimiento informado para la fase 2 del estudio, en el cual se explicó el objetivo y propósito del estudio, el permiso para grabar la entrevista y las posibles consecuencias de su participación y el retiro voluntario que podía efectuar en 
cualquier momento. De esta forma los participaron autorizaron participar en el estudio y contaron libremente sus experiencias. Al tratarse de una investigación sin riesgos, se garantizó a su vez la confidencialidad de los datos obtenidos, advirtiendo a los informantes que en ningún momento aparecería su nombre o ningún dato que pudiera identificarlo durante la exposición de las conclusiones y resultados.

\section{RESULTADOS}

A continuación, se presentan los resultados de cada una de las fases en las cuales se desarrolló el presente estudio.

1. Revisión documental de los syllabus del programa y proyecto educativo del programa de Enfermería (PEP).

Se analizaron un total 32 syllabus de la siguiente manera: 12 del área básica, 11 del área profesional y $9 \mathrm{el}$ área socio-humanística. Estos fueron elegidos de acuerdo a los siguientes criterios: asignaturas que hacían parte del núcleo disciplinar de manera intencional o buscando aquellos syllabus donde más pudiera visibilizarse el concepto de cuidado. Así mismo, se analizó el PEP en sus diferentes componentes encontrándose los aspectos que se presentan a continuación:

1.1. Proyecto educativo del programa de Enfermería (PEP). Desde este documento, el programa reconoce el cuidado de enfermería como el ser de la disciplina y asume que está constituido por el "conjunto de intervenciones, surgidas de la interrelación con el sujeto de cuidado, basadas en el conocimiento científico, técnico y disciplinar, dirigidas a promover y/o recuperar la salud, prevenir la enfermedad y sus complicaciones o favorecer los estilos de vida saludables y con ello, propender por la calidad de vida de quienes participan recíprocamente en esta relación de proximidad" (5:10/28).

El cuidado de enfermería aparece enmarcado en un concepto multidimensional que ocurre en diferentes entornos de complejidad, con unos principios éticos, estéticos y morales integrados en el sujeto de cuidado de estudio. El estudiante del programa, adquiere un compromiso con un cuidado de enfermería enmarcado en la cultura de la vida, su calidad y su sentido desde un enfoque biopsicosocial, cultural y espiritual, este último un componente específico incluido desde este programa de formación que aporta un valor adicional a la concepción de cuidado. El abordaje biopsicosocial y cultural está completamente articulado y coherente con la propuesta institucional y se encuentra evidente en apartados como misión, visión, perfiles, currículo y plan de estudio.

Finalmente aparece el concepto de "gestionar el cuidado" que también se manifiesta en los documentos en aparta como la misión, visión, currículo, perfiles y objetivos de manera reiterativa.

“...con capacidad para proporcionar y gestionar el cuidado de enfermería, fomentar la producción y trasferencia del conocimiento dentro de un marco de derechos en salud (...)" $(5: 1 / 14)$.

En este sentido, es importante considerar que para este programa "la gestión es un proceso humano y social, lo que implica la articulación de los principios administrativos con el cuerpo de conocimientos propios de la disciplina para plantear soluciones de mejora a las diferentes problemáticas del sector salud" (10).

1.2. Syllabus del programa. Después del análisis de los 32 syllabus enmarcados en las tres áreas del programa (básica, profesional y socio-humanística) se encontraron particularidades frente al abordaje del concepto de cuidado, en las cuales cada una aporta significativamente a la formación de los estudiantes.

En el área básica el cuidado se ve enmarcado desde el abordaje de situaciones de salud-enfermedad y como proceso de apoyo de la práctica de enfermería en escenarios reales, tal como se presentan en los relatos que se presentan a continuación.

"En una primera parte se estudian los procedimientos descriptivos y conocimientos básicos lográndolo aplicar en escenarios reales de la Enfermería, describiendo las variables correspondientes a la morbimortalidad y aquellas de interés (...)”. (3:28/5).

"En la asignatura se estudian los aspectos más relevantes que debe enfrentar el futuro profesional de enfermería en relación al cuidado de pacientes con enfermedades infecciosas (...)" (3:1/2).

Los 12 syllabus del área básica buscan aportar a la formación integral de los estudiantes, haciéndose manifiesto especialmente en los objetivos de las dimensiones humana, integración y compromiso; sin embargo, si bien es cierto que el concepto de cuidado está presente en varios de los syllabus de esta área, no es tan explícito en la revisión de documental de los mismos.

Por otro lado, en el área profesional, el cuidado de enfermería se encuentra de manera explícita en los contenidos, justificación, objetivos y algunas de las actividades consignadas en los syllabus. Se hace referencia a la gerencia o gestión del cuidado, el enfoque biopsicosocial-cultural y espiritual, el cuidado integral, las habilidades y competencias para el cuidado y el proceso de atención de enfermería (PAE) como se presenta en los siguientes apartados:

Asignatura teórico práctica con enfoque biopsicosocial 
, cultural y espiritual a individuos y grupos sometidos a procedimientos quirúrgicos que le ofrece al estudiante las herramientas para poder brindar cuidado de enfermería integral" (2:37/5).

"Se hace énfasis particular a dos aspectos: la gerencia del cuidado y la aplicación del proceso de enfermería en la atención de los pacientes. Los demás se enfocan en la gestión del personal, desarrollo de proyectos de gestión, realización de procedimientos y práctica del rol profesional" (2:2/2-16).

En los syllabus del área profesional, es donde más se encuentra presente el concepto del cuidado de enfermería. En algunos de ellos, aparece vinculado a modelos de enfermería como el de Promoción de la Salud de Nola J. Pender o el de Maryory Gordon. Así mismo, se encuentra que éste concepto está presente como intervenciones realizadas en respuesta a necesidades de situaciones de salud específicas de las personas y muchas de ellas, integradas al proceso de atención de enfermería:

"Cuidados de enfermería relacionados con las necesidades espirituales, la agonía y la muerte" (2:62/8).

"Plantear intervenciones de cuidado aplicando el proceso de atención de enfermería" (2:53/7).

Finalmente, en los 9 syllabus del área socio-humanística, el cuidado está enmarcado en el proceso de atención de enfermería y en el enfoque biopsicosocial, cultural y en algunos de ellos en el espiritual (tal como lo propone el PEP del programa). Esto se evidencia en la justificación de las asignaturas y los objetivos de conocimientos fundamentales, compromiso y aplicación. Aunque el concepto de cuidado está presente de manera explícita en algunos de los syllabus, no es constante en la mayoría de ellos.

"Bajo el enfoque biopsicosocial, cultural y espiritual, se espera que el estudiante en este curso identifique $y$ comprenda las diferencias individuales del comportamiento del individuo y su familia". (4:19/9).

"Cuidado de enfermería en las principales situaciones de salud en cada una de las etapas de Desarrollo". (4:2/2).

De esta parte de análisis documental, podemos decir que el concepto del cuidado está presente tanto en el PEP como en los syllabus de las diferentes áreas, sin embrago si tratamos de consolidar cual es el común denominador o lo que significa el cuidado en todos los documentos y la manera en que se aplica, falta integración, es difícil encontrar un significado común, una apuesta institucional común que se evidencie en cada asignatura.

$\mathrm{Al}$ interior de los syllabus encontramos que falta también relación y articulación en todo el documento, ya cuando aparece, se evidencia en forma más clara al principio, o sea en justificaciones y objetivos, pero se va difuminando o diluyendo al tratar de concretizarlo en las actividades y contenidos.
2. Significado del cuidado para estudiantes de último año del programa de Enfermería. En este proceso participaron de manera voluntaria un total de 15 estudiantes. Las categorías emergentes fueron las siguientes: cuidado con enfoque biopsicosocial situado durante los procesos salud-enfermedad, cuidado holístico, conocimientos teórico-prácticos del cuidado.

De la primera categoría cuidado con enfoque biopsicosocial situado durante los procesos salud-enfermedad, emergen dos subcategorías: la primera es la de satisfacción de necesidades de la persona y la otra es la de integrar y ver más allá. Dentro de la primera subcategoría de satisfacción de necesidades de la persona, el estudiante de último año del programa hace referencia directa al cuidado que realiza el profesional para suplir las necesidades de una persona que se encuentra enfrentando una condición de salud específica. El enfoque biopsicosocial surge de manera recurrente en las narraciones encontradas en esta subcategoría, como algo que dio forma a las concepciones sobre el cuidado de enfermería que se formó en los estudiantes desde el primer semestre, como se presenta a continuación:

"Durante mi proceso de formación como estudiante de enfermería, desde el inicio me hablan de un cuidado biopsicosocial, desde primer semestre sabemos que tenemos que tener un enfoque multidimensional y es por eso la concepción que tengo del cuidado según la universidad". (E:7/G: F/R:2).

Además, se puede evidenciar que los estudiantes conciben que el cuidado es multidimensional que contempla lo biológico, lo psicológico, lo social, lo cultural y lo espiritual; que se encuentran presenten en una relación directa y personal con un individuo y que busca cubrir las necesidades de la persona, como ser humano, lo que involucra su entorno, su familia y su rol en la sociedad como se encuentra presente en los siguientes relatos: "Es un enfoque biopsicosocial, o sea, que no solo es enfocarse en el individuo o la patología, sino en las necesidades de él como persona, como ser humano, y todo lo que involucra su entorno, su familia y su rol en la sociedad" (E:3/G: F/R: 8).

Del mismo modo la disciplina enfermera, profundiza en el cuidado del ser humano y del entorno como componentes esenciales de la salud. Desde estas líneas se analizan los aspectos psicosociales que permiten orientar las acciones de enfermería hacia el autocuidado de los pacientes y su familia para describir, explicar y comprender el comportamiento humano, no solo en la enfermedad, sino en aspectos como calidad de vida, adaptación al entorno y el desarrollo de recursos para afrontar las distintas situaciones y alcanzar el bienestar (11).

"El cuidado para mi puede manifestarse como todas las acciones que se hacen para mantener la calidad de vida de una persona, satisfaciendo todas sus necesidades, para así permitir su ritmo de vida normal" (E:5/G: F/R:1). 
Dentro de la segunda subcategoría encontrada, integrar y ver más allá, el estudiante hace referencia directa al cuidado con una perspectiva que busca incorporar los conocimientos y aprendizajes adquiridos en semestres anteriores en la práctica asistencial, no desde un enfoque reduccionista sino más amplio del ser humano.

"Ya tu brindas un cuidado asistencial, o sea, ya lo que viste a lo largo de la carrera lo integras y aparte que no te estás enfocando solo en cómo dar el medicamento, como en la patología, sino ya ves un poco más allá de la humanización en todos los servicios en los que estés, entonces creo que el cuidado abarca mucho eso, más que la administración del medicamento, el estudio de una patología todo eso, mmm...es ver más allá de la persona" (E:3/ G:F/R:4).

En la segunda categoría de los estudiantes se encuentra la del cuidado con enfoque holístico, en la cual emergieron dos subcategorías que fueron: cuidado humanístico y vocación para el cuidado. En la subcategoría del cuidado con enfoque holístico, los estudiantes pueden evidenciar el holismo desde sus prácticas de cuidado al intentar involucrar todas las dimensiones del ser humano en el momento de brindar cuidados. De acuerdo a lo encontrado en algunos relatos, los estudiantes tratan de centrar su cuidado en el ser humano desde un aspecto que integra todas las variables que afectan directamente el bienestar, ya que se hace énfasis en la inclusión de aspectos psicológicos y sociales, además de los biológicos en el momento de brindar la atención al individuo, considerando esto como factores que también inciden en la salud del paciente. También se encuentra que el estudiante establece una clara relación entre el cuidado de enfermería y una perspectiva humanística que le otorga significado a su práctica. Al respecto encontramos este relato:

\section{"El cuidado integra varias partes del individuo en un modelo holístico; es la base fundamental como profesionales de enfermería, también creo que es una necesidad multidimensional de todas las personas en todos los momentos del ciclo vital, aunque en distintos grados en los cuales nosotros como profesionales de enfermería desempeñamos alguna actividad ya sea para tratar o prevenir alguna enfermedad que esté padeciendo o bien la asistencia que le proporcionamos al usuario" (E:7/G: F/R:1).}

Finalmente, en la subcategoría de vocación para el cuidado, los estudiantes la describen como algo que viene del interior de cada persona aportando o dejando una marca en quien se cuida; es algo que es propio de quien estudia carreras como enfermería y que tiene un sentido de valores altruistas que se consolidan durante el proceso formativo.

"Durante mi proceso formativo, donde me enseñaron el ejercicio del cuidado fue durante la práctica profesional donde tuve mi primer contacto con el individuo y pude determinar muchas características importantes que parten de cuestionamientos como: ¿qué voy a hacer? ¿Cómo voy a cuidar?, considero que a partir de éstos cuestionamientos nace el cuidado. El cuidado para mi es vocación" (E:7/G: F/R:10).
La última categoría emergente en los estudiantes fue conocimientos teórico-prácticos del cuidado. Esta consideró dos subcategorías como la brecha entre la academia y la práctica y aportes de la teoría y la práctica. En la primera subcategoría de brecha entre la academia y la práctica, los estudiantes hacen referencia a los cambios en la concepción que tienen sobre el cuidado y lo que encuentran cuando salen a la práctica, esto mediado por los conocimientos y los vacíos que tienen hasta ese momento, e incluso las dificultades para aplicar el cuidado que "debería hacerse", tal como se presenta en los siguientes relatos:

Pues, yo pienso que uno siempre cuando ya va a salir al medio laboral, siente que tiene muchísimos vacíos. Ehh... icreo que es normal! (E:1/G: F/R:8).

"Uno en la práctica sabe que hay momentos donde deberían hacerse muchas cosas para cuidar, pero a veces el medio no lo permite, hay poco tiempo, tienes presiones... uno se da cuenta que ya la cosa es diferente" (E:4/G: F/R:20).

En la segunda subcategoría de aportes de la teoría y la práctica, los estudiantes muestran cómo el cuidado de enfermería ha estado presente desde el primer hasta el último semestre. También se evidencia el conocimiento que tienen los estudiantes del uso de modelos teóricos de enfermería como fundamento para orientar el cuidado de enfermería reconociendo su uso de forma diferente en cada caso y finalmente los estudiantes perciben que en la teoría aprendieron "el deber hacer" y en la práctica "cómo hacerlo" desde la adquisición de habilidades personales y profesionales.

"desde el primer semestre hasta el décimo siempre estuvo la parte del cuidado que es la esencia de nuestra profesión" (E: 5/ G: f/ R: 3).

"Yo creo que, si estas teorías no existieran, no existiría la esencia de la enfermería. Es importante conocer más a fondo los modelos como los de Virginia o de Orem, es decir tener en cuenta varias teoristas para poder ver diferentes maneras para abordar al paciente, y si ellas no hubieran planteado esas teorías no habría sustento científico para brindar cuidado" (E:6/G:F/R:17).

"Marjory Gordon con sus once patrones funcionales y otras, son un aporte grande a la enfermería y da herramientas para poder brindar cuidado. Pero si vemos, lo que aprendemos de esto es muy superficial. Digamos que una de las cosas por mejorar en la Facultad sería esa" (E:7/G:F/R:9).

3. Significado del cuidado para docentes del programa de Enfermería. En esta parte de la investigación se diseñó una guía de preguntas, basados en la revisión de la literatura $\mathrm{y}$ en los resultados obtenidos con las entrevistas a los estudiantes. Se buscó que participaran docentes con dos años de antigüedad como mínimo en el programa de enfermería, que tuvieran contacto directo con los estudiantes y que quisieran participar voluntariamente en el estudio. Se realizaron en total 12 
entrevistas en profundidad y se consideró que pertenecieran a las tres áreas de la estructura organizacional de la Facultad (básica, profesional y sociohumanística). Los resultados de esta fase evidencian tres categorías finales: cuidado desde una concepción individual vs. cuidado de enfermería, un cuidado que privilegia el componente físico, poniéndose de acuerdo para integrar el cuidado: propuestas para la enseñanza y formación del cuidado.

Cuidado desde una concepción individual vs. cuidado de enfermería es la primera categoría desde la cual se desprenden las siguientes temáticas: origen del cuidado, a quién pertenece el cuidado, a quién se cuida, definición de cuidado, objetivos o fines del cuidado, cualidades de quien cuida, características del cuidado, de qué se cuida.

Origen, pertenencia y sujeto/objeto del cuidado. El cuidado para los profesores participantes en el estudio se presenta como algo que tiene un origen natural y que es propio para todos los seres vivos; que surge de una necesidad y que posiblemente podría desarrollarse mejor en las mujeres. Frente al objeto o sujeto de cuidado, los profesores mencionan que se cuida la familia, las personas, la comunidad y los grupos. También se cuida los animales, la naturaleza, el entorno, los compañeros de trabajo y a los estudiantes. Frente al cuidado de los estudiantes, los participantes expresan que cuidan de los estudiantes buscando que sean mejores profesionales, apoyándolos académicamente para que les vaya mejor y buscando su bienestar.

"es un acto y creo que es innato en el ser humano, y sobre todo en las mujeres pienso que se desarrolla un poco más, pero hombres y mujeres pueden cuidar por igual, porque es un acto natural, innato de proteger o de generarle bienestar a las personas de su alrededor" (1:828-1:1101).

Significado del cuidado y objetivos o fines del cuidado. Para los participantes en la investigación, el cuidado tiene un componente que podría denominarse como ontológico y otro más de tipo pragmático. Desde lo ontológico, el cuidado es un sentimiento, una disposición, una responsabilidad y una necesidad. Desde lo pragmático es hacer que las cosas funcionen bien.

Características de quien cuida y las características que son propias de su cuidado. Según los participantes, quien cuida debe escuchar, hablar, tener conocimientos y destrezas. Mostrar interés por el otro y preocupación, tal como lo muestran los siguientes apartes:

"el cuidado para mi es...es el acto de proteger o de generarle bienestar a una persona, un grupo o una comunidad" (1:549-1:666).

Origen y sujeto/objeto del cuidado de enfermería: Los participantes en el estudio indicaron que el cuidado de enfermería es aprendido de dos maneras: durante el pregrado y a través de la experiencia profesional.
“... yo creo que aprendi el cuidado de enfermería fue durante mi formación profesional... ya, pero de... durante mi formación profesional lo aprendí de una manera...implícita. Porque en esa época no se hablaba, cuando yo estudie, hace muchos años no se hablaba de cuidado" (4:496-4:769).

Significado del cuidado y objetivos o fines del cuidado de enfermería. El cuidado de enfermería para los participantes del estudio, se relaciona con el cuidado que realiza un profesional, como apoyo, ofreciendo atenciones, realizando actividades y acciones con un sentido, propósito o dirección, así como la identificación de necesidades para mantener la salud o mejorar la enfermedad. Por otra parte, los participantes del presente estudio, también identificaron objetivos o fines del cuidado de enfermería y dentro de ellos se encuentran: Alcanzar bienestar, solucionar una situación de salud o una necesidad, solventar una actividad que el paciente ya no puede hacer por sí mismo, identificar unas necesidades de cuidado, prevenir enfermedades, mejorar el estado de salud, suplir una necesidad o una respuesta a una necesidad de vida (física, emocional y trascendental); es preservar la especie.

Cualidades de quien cuida y características del cuidado de enfermería. Para los participantes del presente estudio, son cualidades de la persona que brinda cuidado de enfermería: mirar sus propias fortalezas y debilidades, preocuparse, sentir empatía, ofrecerlo sin condición, estar capacitada. De otra manera, los participantes expresaron además algunas características o atributos del cuidado de enfermería en aspectos como: el contacto con los pacientes, el estar con ellos, es organizado, profundo, tiene unos principios y lineamientos que orientan la práctica, tiene una filosofía que lo sustenta, es científico, tiene un cuerpo de conocimientos propio, debe tener un seguimiento, ser evaluado, retroalimentado, tener un consenso, es tangible, visible, medible. Los siguientes fragmentos manifestados por los participantes describen esas cualidades y características:

"Como enfermera, sí considero que nosotros tenemos un cuerpo de conocimiento propio, lo cual nos hace que seamos profesionales y sí requiere de conocimiento científico, que es un poco lo que diferencia al otro cuidado (1:2801-2:107)".

"El concepto de cuidado de enfermería es mucho más profundo, es organizado, tiene unos principios y tiene unos lineamientos que orientan para su práctica, y basados en estos y en una filosofía... eh... las que estamos en esta profesión, brindamos esa acción de cuidado, cumpliendo esos requerimientos y esos lineamientos que nos da una disciplina (2:2844-2:3190)".

Se menciona un resumen de las principales percepciones sobre el cuidado y el cuidado de enfermería de los profesores: Ver Tabla 1.

Un cuidado que privilegia el componente físico es la segunda categoría que emerge de las entrevistas con los profesores, quienes evidencian que para muchos estudiantes el valor de 
1 procedimiento físico es superior a los cuidados psicosociales, culturales y espirituales que tiene el individuo en un momento determinado.

Tabla 1: Conceptualización del cuidado y el cuidado de enfermería por los docentes

\begin{tabular}{|c|c|c|}
\hline $\begin{array}{l}\text { Característica } \\
\text { - Concepto } \\
\end{array}$ & Cuidado & Cuidado de enfermería \\
\hline Origen & $\begin{array}{l}\text { Es innato y natural. } \\
\text { Es algo cotidiano. }\end{array}$ & $\begin{array}{c}\text { Es el pregrado o la } \\
\text { academia, la experiencia } \\
\text { personal o profesional } \\
\text { donde se da. } \\
\end{array}$ \\
\hline $\begin{array}{r}\text { A quién } \\
\text { pertenece el } \\
\text { cuidado }\end{array}$ & $\begin{array}{l}\text { No es exclusivo de los } \\
\text { seres humanos, sino } \\
\text { que los seres vivos en } \\
\text { general lo tienen. }\end{array}$ & A enfermeras y enfermeros. \\
\hline $\begin{array}{l}\text { Característica } \\
\text { - Concepto }\end{array}$ & Cuidado & Cuidado de enfermería \\
\hline $\begin{array}{r}\text { A quién se } \\
\text { cuida }\end{array}$ & $\begin{array}{l}\text { Se cuida a la familia, } \\
\text { las personas, la } \\
\text { comunidad y los } \\
\text { grupos. También se } \\
\text { cuida a los animales, } \\
\text { a la naturaleza, al } \\
\text { entorno, a los } \\
\text { compañeros de } \\
\text { trabajo, a los } \\
\text { estudiantes. }\end{array}$ & $\begin{array}{c}\text { A los pacientes, las } \\
\text { personas y la comunidad. }\end{array}$ \\
\hline $\begin{array}{r}\text { El cuidado } \\
\text { es... }\end{array}$ & $\begin{array}{l}\text { Un sentimiento, } \\
\text { brindar apoyo, } \\
\text { acompañar, es un } \\
\text { estado de disposición, } \\
\text { un acto de "hacer", } \\
\text { una ayuda integral, } \\
\text { una responsabilidad, } \\
\text { una adaptación } \\
\text { filogenética, una } \\
\text { necesidad. }\end{array}$ & $\begin{array}{c}\text { El cuidado profesional, es } \\
\text { apoyo o apoyo al enfermo, } \\
\text { es mantener la salud y } \\
\text { mejorar el pronóstico de la } \\
\text { enfermedad. Implica } \\
\text { atenciones, actividades o } \\
\text { acciones (como } \\
\text { administración de } \\
\text { medicamentos, } \\
\text { tratamientos o } \\
\text { procedimientos) de } \\
\text { acuerdo a la enfermedado } \\
\text { la situación. }\end{array}$ \\
\hline $\begin{array}{r}\text { Objetivo o } \\
\text { fines }\end{array}$ & $\begin{array}{l}\text { Protección, } \\
\text { seguridad, bienestar, } \\
\text { funcionar bien, } \\
\text { satisfacer } \\
\text { necesidades, hacer } \\
\text { mejores profesionales } \\
\text { a los estudiantes. }\end{array}$ & $\begin{array}{l}\text { Bienestar, solucionar una } \\
\text { situación de salud o una } \\
\text { necesidad, solventar una } \\
\text { actividad que el paciente } \\
\text { ya no puede hacer por si } \\
\text { mismo, identificar unas } \\
\text { necesidades de cuidado. }\end{array}$ \\
\hline $\begin{array}{r}\text { Cualidades de } \\
\text { quien cuida }\end{array}$ & $\begin{array}{l}\text { Escuchar, hablar, } \\
\text { tener conocimientos y } \\
\text { destrezas. Mostrar } \\
\text { interés (preguntar) y } \\
\text { preocupación. }\end{array}$ & $\begin{array}{c}\text { Mira sus propias fortalezas } \\
\text { y debilidades, se preocupa, } \\
\text { siente empatía, se ofrece } \\
\text { sin condición, debe tener } \\
\text { una capacitación. }\end{array}$ \\
\hline $\begin{array}{r}\text { De qué se } \\
\text { cuida }\end{array}$ & $\begin{array}{l}\text { Se cuida del peligro, } \\
\text { de las amenazas, de } \\
\text { las enfermedades. }\end{array}$ & Las enfermedades \\
\hline
\end{tabular}

El estudiante solamente reconoce el cuidado físico. Desconoce las otras dimensiones: la parte social, no la incluye; la parte espiritual, no la reconoce; la parte cultural, lo asume, pero desde...desde mi percepción, desde un enfoque diferente; o sea no tiene en cuenta que los arraigos culturales hacen que las personas obren y entiendan de determinada forma. No lo veo presente en la mayor parte de los estudiantes no lo veo. (2:8/1918).

"Entonces para el estudiante es mucho más beneficioso decir que en la práctica canalizó diez venas, a decir, mire, tuve una intervención en crisis a una señora que estaba en depresión (...)”. (4:7/275).

Propuestas para la enseñanza y formación del cuidado es la última categoría que emerge del análisis de lo manifestado por los docentes, en ella se encontraron las siguientes subcategorías: ponerse de acuerdo para integrar el cuidado, ser más flexibles y visionarios, fortalecer la formación como docentes y con vocaciones, mejorar el número de estudiantes por docente, participar en escenarios políticos $y$ empoderamiento de la Enfermería.

Ponerse de acuerdo para integrar el cuidado. Los docentes mencionan que, tanto para los estudiantes como para ellos, es importante integrar las cosas y mencionan que es importante ponerse de acuerdo. Algunos manifiestan que el cuidado tiene su lenguaje y que hay que buscar acercamientos.

"Yo parto del hecho, conociendo el currículo del programa que ellos lo traen, pero no lo tienen claro y no lo integran. Para mi es un poco difícil porque tratar de hacerle ver $\boldsymbol{y}$ visibilizar en el estudiante todas las dimensiones del sujeto desde el acto del cuidado, no es fácil" (2:4/425).

"Entonces que nos dijeran cuál es el leguaje, como usted tiene un paciente, con tal no sé... algo de enfermería, entonces cuál sería el "cuidado" que usted le daría. Porque los de las básicas estamos per...o sea, nosotros ahí como a ciegas más o menos" $(10: 9 / 2115)$.

Para fortalecer la articulación entre el cuidado con el enfoque biopsicosocial, cultural y espiritual que se propone en el programa de enfermería, recomiendan hacer más evidente los aspectos centrales de enfermería en los syllabus del área básica y promover un dialogo conjunto entre las tres áreas. En la facultad se tienen tres áreas administrativas: básica, profesional y socio-humanística y sobre ellas se fundamentan también los conocimientos que se imparten a los estudiantes del programa de enfermería. Sin embargo, los docentes participantes piensan que puede existir una mayor articulación de las tres áreas, que puedan incluir la formación en el cuidado de enfermería de manera transversal.

"Primero que todo debemos entender que las áreas...las otras dos áreas que acompañan el área profesional son ...son los dos pies que soportan la formación del estudiante. Y así como uno tiene los pies, que hacen parte de su cuerpo, no pueden estar desligados. No habría coordinación, no habría motricidad, no habría... tienen que estar integradas. Y eso tiene que estar desde el primer semestre”. (2:6/15)

"Pero eso es de dialogar entre profesores, no solo de la profesional, sino de básica, de socio humanística, pero mientras estemos divididos no hacemos nada" (4:9/2014).

Ser más flexibles y visionarios. Esta subcategoría muestra como los docentes proponen un cambio que tienda a generar un tipo de pensamiento más flexible y que dé paso a nuevas y creativas ideas que tiendan al mejoramiento de la profesión y de la enseñanza de la Enfermería. Se propone el aprender aceptar los 
cambios, pensar en lo que los estudiantes les gustaría hacer y escuchar lo que ellos proponen y hacer ajustes a partir de un dialogo conjunto entre los docentes.

Nosotras tenemos que ser muy visionarias y tenemos que ser mucho más flexibles con todo esto, mejor dicho, aceptar los cambios ¿no? y a enfrentarnos también a esos cambios. Entonces nosotros somos demasiado cuadriculados, entonces pues, asi lo hemos hecho durante veinte años, entonces no se puede cambiar" (9:15/1916).

Fortalecer la formación como docentes y formar con vocación. El docente universitario, tradicionalmente enseña de la misma forma en la que aprendió. Es decir, todas las estrategias didácticas y pedagógicas que utilizaron sus docentes para su aprendizaje, serán utilizadas por el mismo en su ejercicio docente. En ese sentido los participantes del estudio evidencian la necesidad de formación en pedagogía, el fortalecimiento de competencias de análisis crítico, de pensamiento crítico y la necesidad de tener vocación para ser docente que se va a ver reflejado en los microcurrículos del programa.

"Muchas veces, y es también muy triste ver, cómo muchas veces nos quedamos solo en trasmitir información y solo le pedimos a los estudiantes... (en la gran mayoría, no siempre ¿no?) Pedimos que se memorice, entonces dejamos como a un lado, como esas competencias de análisis crítico, de pensamiento crítico, analítico que debería fomentarse actualmente en los estudiantes" (1:8/2126).

"También considero que una de las causas es el mismo desconocimiento y la poca formación que tenemos en pedagogía $\mathrm{y}$ en educación y eso dificulta un poquito el aprendizaje (...)" (1:19/15).

Número de estudiantes por docente. En la Universidad, además del modelo biopsicosocial, en materia de pedagogía es abanderado por el aprendizaje significativo; el cual, en general, no habla de enseñanza, sino de aprendizaje y propende por un estudiante que sea protagonista de ese aprendizaje. En este sentido las propuestas generales de los docentes apuntan a reflexionar sobre el número de estudiantes que se encuentran a cargo de cada docente tanto en teoría, en la práctica y también en los grupos de investigación formativa.

Participación en escenarios políticos y empoderamiento de la enfermería. Los docentes participantes hacen un llamado a la necesidad de incluir en los estudiantes de enfermería la formación en política y participación ciudadana con un mayor énfasis. Este aspecto emerge como algo de consciencia profesional, sobre la obligación que tienen los profesionales de enfermería de contribuir con la solución de las principales problemáticas de salud en el país y de la práctica de enfermería respecto a sus condiciones actuales, en escenarios de la política pública que generan temor o miedo. Así mismo, los participantes reconocen que el empoderamiento ganado por las enfermeras está respaldado por el conocimiento, experiencia y compromiso profesional. En este apartado se pone en evidencia la necesidad que tienen los profesionales de enfermería de empoderarse de su saber disciplinar y del cuidado de enfermería para de esta manera, poderlo transmitir de manera motivada a los estudiantes en formación.

“Una propuesta para mejorar la ... el ejercicio del cuidado de enfermería en el país, es que todas las facultades deberían tener una asignatura de componente político, porque a los enfermeros nos da miedo estar en la política o hacer normas, o bueno sí, políticas. ¡Nos da miedo!” (5/8:129).

"Yo creo que haría falta empoderarnos más. $Y$ verdaderamente, si yo digo, seamos lideres, pues el primero en ser líder sea usted (...)” (8:4/734).

"Desde la parte de la motivación del profesional frente al cuidado. Porque si no hay motivación, yo sigo haciendo las cosas de la misma manera, mal o bien, pero...y no me motivaría de pronto a investigar, a darle respuesta a mis dudas (...)" $(2: 8 / 311)$

Los profesores participantes mencionan la importancia de seguir fortalecimiento del cuidado, su sustento y los aspectos disciplinares de enfermería al interior de la facultad y de manera continua; a través de encuentros entre docentes y talleres en los que todos puedan llegar a consensos y hablar el mismo idioma:

"Yo pienso que lo primero que hay que hacer, es hacer como unos encuentros de profesores donde se hable de cuidado, donde se compartan experiencias, se compartan conceptos, ideas, hasta que todos lleguen a un consenso de qué es el cuidado" (6:9/978).

"En términos del cuidado de enfermería creo que nos hace muchísima falta muchísima información sobre teorías de cuidado y sobre el cuidado en enfermería. Si yo me pongo hacer un análisis crítico de la práctica que hacemos cada uno de nosotros, muy pocas veces mientras estamos en el espacio de práctica lo recordamos a los estudiantes... ustedes qué teoría de enfermería ven aquí, pueden aplicar o dónde estoy parada yo" (12:6/2805).

\section{DISCUSIÓN}

Los resultados del estudio en sus dos fases muestran la experiencia de un programa de enfermería en la formación en el cuidado de enfermería de sus estudiantes. La revisión documental de la fase 1 evidencia que el concepto de cuidado de enfermería planteado desde el PEP, guarda relación con lo referido en la literatura, al hacer referencia a un concepto multidimensional que involucra aspectos de calidad, principios éticos, estéticos y morales (12). Que además involucra una serie de intervenciones basadas en conocimientos científicos, técnicos y disciplinares que promueven la salud, su recuperación, la prevención de la enfermedad y favorecen los estilos de vida saludables; a través de una interrelación recíproca con el individuo (13), con el objetivo de alcanzar su calidad de vida (14). El concepto del programa añade además que este cuidado de enfermería involucra con compromiso con 
una cultura de la vida, su calidad y su sentido desde un enfoque biopsicosocial, cultural y espiritual.

Al reflexionar sobre la definición anterior, es importante considerar que Enfermería ha luchado por conformar un cuerpo de conocimientos propio que le permitan un trabajo independiente y autónomo del modelo biomédico predominante. Esto es posible con articulación de las teorías generales, la investigación (desde los paradigmas naturalista, relativista e interpretativo) (15), la práctica de enfermería basada en la evidencia (16) y el reconocimiento de los saberes propios de enfermería, que se hacen evidentes en los patrones de conocimiento de enfermería. Como lo manifiesta Pina "estos conocimientos tienen naturaleza diversificada y se agrupan en patrones, que son: empírico, (conocimiento factual, descriptivo y verificable o evidencias científicas); ético, (comportando valores, normas y principios); estético, (el arte que es trasmitida por medio de la intuición, sensibilidad y técnica; personal, de la autenticidad de la relación reciproca con el otro) ; reflexivo, creado en la reflexión en la acción, la reflexión sobre la acción, reflexión sobre la reflexión en la acción; sociopolítico y de emancipación, del conocimiento de la diversidad de los contexto y del ambiente"(17), que le dan sentido al conocimiento adquirido desde la práctica de enfermera.

De la misma forma, la perspectiva de reciprocidad propuesta desde la definición de cuidado de enfermería del PEP, guarda relación con la visión de reciprocidad o interactiva-integrativa que propone Fawcett (13), la cual plantea que "Los seres humanos son partes holísticas vistas sólo en el conjunto del contexto"; "los seres humanos son activos, su interacción con otros seres humanos y con el medio ambiente es recíproca"; lo que implica la posibilidad innata del ser humano de relacionarse entre sí, con otros seres humanos y a su vez con el medio ambiente. Lo que genera interacciones necesarias para el desarrollo de sí mismo y de otros en su entorno. "El cambio es una función con múltiples factores. Es probabilística y tal vez continua o tal vez solo por sobrevivir". Fawcett explica como las experiencias en la vida de las personas, las motiva a generar cambios, que deben proyectarse en su vida futura en forma positiva, garantizando el bienestar propio y de las personas cercanas. Finalmente, se evidencian cambios paradigmáticos de pensamiento, en donde la realidad y la ciencia no sólo es cuantificable, sino que es multidimensional y se puede estudiar desde distintos enfoques del conocimiento. En este sentido, se reconoce que, para el programa de enfermería, el sujeto de cuidado es un actor activo en su proceso de salud y en constante interacción con el contexto que le rodea, por lo cual puede mostrar cambios de manera constante.

Frente al enfoque biopsicosocial, cultural y espiritual, las dimensiones tienen significado en la medida en que se establece una estrecha relación entre el responsable del cuidado de la salud, la persona, la comunidad, la familia y la sociedad, para así responder y satisfacer todas aquellas necesidades de los individuos dentro de un contexto global y transdisciplinario (18), lo que implica la participación de diferentes disciplinas y áreas de conocimiento para abordar la totalidad de la persona desde el cuidado es necesario.Se reconoce además que esta concepción de cuidado de enfermería, se gestiona y organiza administrativamente en las áreas básica, socio-humanística y profesional. El cuidado de la persona es el centro del área profesional, el cual se produce con la integración de los sujetos de cuidado en sus diferentes etapas del ciclo vital, que aporta el conocimiento disciplinar, así como las herramientas administrativas y gerenciales al estudiante. Frente a este aspecto, Meleis establece que "la gestión de los cuidados se ejerce no solamente con los recursos que dan las teorías de la administración, sino también con las relaciones y concepciones propias de la disciplina de enfermería" (19). Igualmente, Morfi define la gestión del cuidado de enfermería como la aplicación de un juicio profesional en la planificación, organización, motivación y control de la provisión de cuidados, oportunos, seguros, integrales, que aseguren la continuidad de la atención y se sustenten en lineamientos estratégicos para obtener como producto final la salud (20). Estas definiciones llevan a pensar que la gestión del cuidado puede ser abordada desde dos aspectos, uno encaminado a la provisión del cuidado directo a la persona, la familia o la comunidad; y el otro hacia el uso de modelos administrativos necesarios para facilitar el cuidado a quienes brindan el cuidado directo.

El cuidado de enfermería por otro lado, es traducido por el área socio-humanística desde el enfoque biopsicosocial, cultural y espiritual, aportándole al estudiante una mirada de las diferentes dimensiones del ser humano. En el caso del área básica, este cuidado no ha sido del todo traducido, debido a que posiblemente no se reconoce, por lo cual los docentes se enfocan en proveer al estudiante de conocimientos específicos de áreas como fisiología, microbiología, patología, farmacología, etc., sin articular los aspectos generales del cuidado de enfermería. Lo anterior, puede evidenciar que es necesario fortalecer este proceso de retroalimentación entre lo que cada una de las áreas aporta al estudiante, no desde la mirada del ser humano como centro, sino del cuidado de la experiencia de la salud de la persona que es el objeto del cuidado de enfermería. Es importante establecer además mecanismos para que tanto el área básica, como el área socio humanística, aporten en mayor grado a la construcción de una formación en el cuidado integral de los estudiantes, con conocimientos de las ciencias básicas y humanísticas suficientes y articuladas.

Surge entonces la pregunta de cuál sería la mejor manera de que el concepto de cuidado de enfermería que se encuentra en el PEP del programa, se gestione en las tres áreas de la Facultad y del programa de enfermería. Esta pregunta requiere ser resuelta en futuros estudios, pero una clave para avanzar en esta área se encuentra en el dialogo y la retroalimentación entre los docentes de las tres áreas de la Facultad, quienes reconocen la necesidad de que "todos hablen el mismo lenguaje" cuando se hable de cuidado y de hacer visible el concepto en mayor grado en los syllabus de las diferentes áreas.

De otra manera, al establecer lo que los docentes comprenden sobre el cuidado y el cuidado de enfermería, reconcomen que el cuidado es algo que pertenece a todos los seres vivientes (21) y que comprende lo cotidiano, se debe cuidar "todo" (22). Cuando hacen mención a su definición y el objetivo del cuidado, su comprensión frente al concepto se da desde "el ser 
del cuidado", reconociendo las características más grandes e ideales que comprende. El fin último es la supervivencia, la protección contra el peligro y dentro de este peligro, están las enfermedades. Cuando se habla del cuidado de enfermería, por otro lado, los docentes reconocen que es algo que involucra directamente a los profesionales de enfermería y que se adquiere en la academia (23) (24). Se cuida a las personas, familias y comunidades y al indagar sobre su definición y objetivo, los docentes lo enfocan en "el hacer del cuidado", es decir en las acciones que realiza el profesional. Se cuida de la persona enferma; sin embargo, es importante reconocer que la enfermedad es solo una expresión de la salud (25).

Boykin y colaboradores, manifiestan que el cuidado es una característica esencial y una expresión del ser humano porque las personas cuidan en virtud de su humanidad (26) (27). El cuidado es además un imperativo moral, porque implica además una disposición personal, una responsabilidad $\mathrm{y}$ una preocupación con otras personas. Es "una forma de fomentar las relaciones con otro ser valioso hacia quien uno tiene un sentido personal de compromiso y responsabilidad" (28). Igualmente, Busquets citada por Carrillo y colaboradores, expresa que "cuidar es acompañar a la persona en sus experiencias de vida. Por ello enfermería trata de planificar la atención desde esta perspectiva" (29). Además de estos aspectos, los docentes recalcan la importancia de cuidar a sus estudiantes, tal y como lo manifiestan Flores y colaboradores (24), quienes consideran que el docente de enfermería debe brindar mayor significado a la relación humanizada e interpersonal con el estudiante y comprenderlo desde su complejidad y dimensiones para poder brindar un cuidado adecuado. Esto incluye el cuidado que realiza el docente de sus estudiantes a cargo.

Frente al cuidado de enfermería en la Facultad, los docentes proponen la necesidad de una mayor formación de los estudiantes en la política (30) (31), teniendo en cuenta los desafíos que enfrenta actualmente Enfermería, no solo en la contribución de las condiciones de salud de la población, sino en las condiciones mismas de la profesión y de su reconocimiento en los roles actuales. Promueven además un mayor liderazgo y empoderamiento, que para ellos se ve relejado en el conocimiento, experiencia y compromiso son su profesión. Así mismo, reconocen la necesidad de seguir fortaleciendo su saber disciplinar y la reflexión al interior de la Facultad frente al cuidado de enfermería. Todos estos aspectos guardan relación con el plan de desarrollo propuesto por la Asociación Colombiana de Facultades de Enfermería (ACOFAEN) en el periodo 2018-2029 (3), es el consenso de la campaña Nursing $\operatorname{Now}(32)$ y de diferentes autores de enfermería (33) (34).

Por otro lado, los docentes mencionen que a los estudiantes de enfermería les falta fortalecer el componente del cuidado de enfermería, les cuesta integrar el cuidado o dan mayor valor a la dimensión física de la persona, que hacen evidente cuando solo mencionan la enfermedad o cuando dan más valor al aprendizaje de un procedimiento al cuidado mismo en la práctica. Al indagar con los estudiantes es evidente la presencia de una mirada biomédica del cuidado, donde quizás las asignaturas con un énfasis biopatológico, por encima de asignaturas que hacen referencia a aspectos menos procedimentales de la atención de la persona. Sin embargo, pese a lo anterior existe un reconocimiento de varios de los aspectos fundamentales del cuidado de enfermería con el del enfoque biopsicosocial, cultural y espiritual que se les inculcó desde el primer semestre en el programa.

En este sentido, puede decirse que los estudiantes de enfermería del programa tienen una concepción básica y general sobre los rasgos más distintivos del cuidado. $\mathrm{Si}$ bien es cierto la resolución de necesidades como parte del cuidado de enfermería es lo que Durán de Villalobos (35) establece como cuidado contractual o de convenio en el cual hay un reconocimiento de parte de quien cuida, de las necesidades y obligaciones del paciente y tiene como fin su beneficio, su compromiso y el reconocimiento de la necesidad de ayuda; lo que indicaría según la autora, es que un cuidado enfocado solamente en la resolución de necesidades se caracterizaría por una relación productiva entre la enfermera y el sujeto de cuidado, en el establecimiento de un acuerdo de confianza y no de reciprocidad, en una relación donde la enfermera sabe más del sujeto de cuidado y lo que requiere, y que por lo tanto no se establece un dialogo conjunto, sino simplemente de responsabilidad frente esas necesidades. Lo que implicaría que hay que seguir trabajando en los estudiantes desde una perspectiva de formación en el cuidado de enfermería con abordajes más integrativos y totales de la persona.

También los estudiantes de enfermería reconocen que para cuidar se requiere de una relación con el sujeto de cuidado y que el cuidado es multidimensional, por lo que se requiere del reconocimiento de las diferentes dimensiones del ser humano para poder proporcionar los cuidados. Además, comprenden que el cuidado es la esencia de la Enfermería y que los modelos y teorías de enfermería dan no solo el sustento científico para cuidar, sino que marcan diferentes formas de abordar al paciente (36).

Teniendo en cuenta todo lo anterior, podría decirse que, de alguna forma, los estudiantes están integrando la gran cantidad de conocimientos que reciben durante su formación y le dan forma a una concepción propia del cuidado de enfermería y su significado. Quizás cuando los docentes manifiestan que los estudiantes se enfocan en la dimensión física o en el procedimiento, lo que hacen los estudiantes es fijar su atención en el mejoramiento de habilidades técnicas para más adelante ejercer acciones de cuidado. Es posible que conforme aumente su experiencia y sus habilidades prácticas, los demás aspectos que se inculcaron desde su formación frente al cuidado de enfermería, empiecen a cobrar sentido, como ocurre en los estudiantes de último año, que han tenido una experiencia de cuidado más independiente gracias a sus prácticas formativas. Una cuestión importante en este aspecto sería cómo ayudar a que los estudiantes puedan integrar más fácilmente los conocimientos frente al cuidado, lo cual requerirá no solo de conocimientos pedagógicos sino del dialogo entre los docentes.

De otra manera, los estudiantes reconocen que existe una brecha entre la teoría y la práctica, y los conocimientos y vacíos 
con los que cuentan, cuando están a punto de graduarse como profesionales de enfermería. Viven la confrontación del" deber ser" y el "cómo hacerlo" en la práctica, lo cual no es ajeno a la situación misma de la enfermería en el país, en la hay una gran brecha entre la investigación, la asistencia y la academia (37). En este sentido lo que perciben los estudiantes, puede ser un reflejo de esta realidad en la que además de la brecha entre esas tres dimensiones; están las relacionadas con las condiciones mismas del sistema de salud, las condiciones de los profesionales en ejercicio, entre otras, que en muchos casos genera un conflicto con los ideales del cuidado y del ejercicio de la enfermería de las que se habla en la academia y que debe trasladarse a la práctica enfermera (38).

Finalmente, los docentes del programa de enfermería proponen algunas estrategias para ser fortalecidas y se relacionan con mejorar el número de estudiantes por docente, al considerar la importancia no solo de enseñar bajo un enfoque biopsicosocial, sino al mismo tiempo bajo el modelo de aprendizaje significativo, que es el modelo pedagógico de la IES del estudio (39). Los docentes también recomiendan aumentar la flexibilidad y ser más visionarios, teniendo en cuenta no solo a la opinión de los demás docentes sino a las propuestas de los propios estudiantes y lo que a ellos les gustaría aprender sobre el cuidado. Así mismo, manifiestan que es importante fortalecer la formación como docentes en pedagogías para la enseñanza y enseñanza de análisis y pensamiento crítico, que contribuya a fortalecer su labor docente en el programa de enfermería. Todo lo anterior, genera la reflexión frente a los aspectos relacionados con la enseñanza del cuidado en el currículo y genera cuatro preguntas "¿Qué enseñar? ¿Cuándo enseñar? ¿Cómo enseñar? y ¿Qué cuándo y cómo evaluar?”. Esta es una reflexión continua y constante para los micro currículos, que debe ajustarse a la luz de los cambios en el contexto y los avances en la enfermería basada en la evidencia.

El cuidado como materia de estudio de enfermería se relaciona con las manifestaciones que han planteado el "saber" y el "quehacer" de la actividad de cuidar a lo largo de los siglos (40). Es reconocido que, en la historia de enfermería, el cuidado ha pasado por diferentes transformaciones, (tal como ocurrió en las etapas: doméstica, vocacional, técnica y profesional), y en su evolución, se evidencia la influencia de la formación y la instrucción frente a un conocimiento construido paulatinamente sobre cómo cuidar a otro. Nightingale por ejemplo, visionó la formación de las enfermeras desde propuestas conceptuales sustentadas con postulados que buscaban generar identidad en la práctica de enfermería, separándose de los conceptos médicos mas no desligándose de los mismos. De esta manera, la formación propuesta por Nightingale buscaba que aun por fuera del contexto hospitalario, la enfermera tuviera herramientas para sustentar su acción y su ejercicio (41).

Actualmente, el desarrollo del concepto sigue transformándose para abrirse paso desde lo profesional al profesionalismo de enfermería y como lo menciona Valdivia y Velandia, "este hecho permite plantearse inquietudes de superación profesional y de realización personal que van en sintonía con el contexto y el nivel alcanzado por la profesión en la práctica y por la disciplina en el conocimiento del cuidado, para poder plantearse de manera responsable ante la sociedad y aportar con iniciativa y creatividad" (40). En este sentido, el aprendizaje del cuidado en el siglo XXI cobra significado e invita a reflexión sobre la manera en que actualmente se viene construyendo.

\section{CONCLUSIONES}

Es importante promover el diálogo entre los diferentes actores con miras a ampliar las reflexiones epistemológicas del cuidado de manera transversal desde un paradigma de la reciprocidad. Así mismo, la integración del enfoque biopsicosocial, cultural y espiritual, debe seguirse fortaleciendo con el fin de que sea comprendido e incorporado integralmente en todas las actividades formativas y de la cotidianidad del programa.

$\mathrm{Si}$ se quiere realmente generar impactos curriculares y pedagógicos, es necesario entender que debemos trabajar por cambiar paradigmas, los mismos que por años nos han anquilosado en formas de pensar estáticas, imitadoras o rígidas, y que, por miedo a lo desconocido, por rutina o por exceso de trabajo, se prefiere simplemente permanecer en la zona de confort, el cual nada aporta a lo que disciplinariamente y conceptualmente necesita nuestra profesión en estos momentos hegemónicos de cambio.

Cada una de las áreas profesional, básica y socio humanística aportan elementos a la formación de los estudiantes en la concepción de cuidado, que son valiosas y deben integrarse de mejor manera para la formación en cuidado de enfermería. El cambio es un proceso en el que se participa en forma activa y con motivación para involucrar a todos aquellos que hacen parte del mismo. Los cambios para el crecimiento y mejoramiento implican pensar diferente y permitir a otros expresar sus pensamientos e ideas frente a las mejores formas de formar a los estudiantes en el cuidado, y eso incluye a los propios estudiantes y a los docentes. Grupos de trabajo con altos grados de motivación y el tiempo necesario para realizar y desarrollar estas propuestas se deben fomentar, para generar reflexiones y aportes significativos para la disciplina y la formación de los profesionales de enfermería. De la misma forma se deben buscar estrategias para que la academia y la práctica de enfermería se unan, buscando alcanzar el "deber ser del cuidado" en la práctica enfermera actual

Conflicto de Interés: Las investigadoras declaran no tener ningún conflicto de interés en la realización del estudio.

\section{REFERENCIAS}

1. Finkelman A, Kenner C. The esence of nursing: knoledge and caring. En: Professional nursing concepts Cmpetencies for quality leadership. 3.a ed. 5 Wall Street: Jones \& Barlett Learning, LLC, an Ascend Learning Company; 2016. p. 4165.

2. Congreso República de Colombia. Ley 911 de 2004 Responsabilidad deontológica para el ejercicio de la profesión de Enfermeria en Colombia [Internet]. 2004. p. 1$15 . \quad$ Disponible en: http://www.mineducacion.gov.co/1621/articles105034_archivo_pdf.pdf 
3. ACOFAEN. Asociación Colombiana de Facultades de Enfermería. Plan de desarrollo 2018 - 2022. Bogotá, Colombia; 2018. p. 1-30.

4. Candido de Oliveira Salvador PT, Vítor AF, Ferreira Júnior MA, Domingues Fernandes MI, Pereira Santos VE. Systematization of teaching nursing care at a technical level: perception of professors. Acta Paul Enferm [Internet]. 2016;29(5):525-33. Disponible en: http://www.scielo.br/pdf/ape/v29n5/en_1982-0194-ape-2905-0525.pdf

5. Rodrigues J, Zeferino MT, Tosoli M. Integrative review about the teaching of the nursing care in mental health. Rev Pesqui Cuid é Fundam Online [Internet]. 2014;6(1):433-49. Disponible

en: http://www.seer.unirio.br/index.php/cuidadofundamental/ar ticle/view/2650/pdf_1109

6. Becerril LC, Gómez BA. Cuidado de enfermería como eje del currículum en la educación profesional: perspectiva de aprendizaje. Rev Horizontes en Salud [Internet]. 2013;5(Enero-Julio):39-59. Disponible en: http://web.uaemex.mx/revistahorizontes/docs/revistas/Vol5 /CUIDADO_DE_ENFERMERIA.pdf

7. Wikström B. Works of art: a complement to theoretical knowledge when teaching nursing care. J Clin Nurs. 2001;10(1):25-32.

8. Gómez OJ, Carrillo GM, Cárdenas DC. Estrategias para el aprendizaje significativo del cuidado humano en enfermería. Arch Med [Internet]. 2017;17:437-44. Disponible en: http://revistasum.umanizales.edu.co/ojs/index.php/archivos medicina/article/view/1986, 20171206.

9. Ministerio de Salud. República de Colombia. Resolución $\mathrm{N}^{\circ}$ 008430. Const Política Colomb [Internet]. 1993; Disponible en:

https://www.minsalud.gov.co/sites/rid/Lists/BibliotecaDigit al/RIDE/DE/DIJ/RESOLUCION-8430-DE-1993.PDF

10. Facultad de Enfermería UEB. Proyecto educativo del programa (PEP). Bogotá: Universidad El Bosque; 2014.

11. Carbelo Baquero B, Romero Llort M, Casas Martínez F, Ruiz Ureña T, Rodríguez de la Parra S. Teoría enfermera. Cult los Cuid [Internet]. 1997;1(2):54-9. Disponible en: http://rua.ua.es/dspace/bitstream/10045/5303/1/CC_02 09 . pdf

12. Ramírez P, Müggenburg C. Relaciones personales entre la enfermera y el paciente. Enfermería Univ [Internet]. 2015;12(3):134-43. Disponible en: http://www.revistaenfermeria.unam.mx/ojs/index.php/enfermeriauniversitaria/ article/view/132

13. Fawcett J, DeSanto-Madeya S. Contemporany Nursing Knoledge. 3rd ed. Philaldelphia: Davis Company; 2013. 3$21 \mathrm{p}$.

14. Cabal E. VE, Guarnizo-Tole M. Enfermería como disciplina. Rev Colomb Enfermería. 2018;6(6):73.

15. Durán de Villalobos MM. La ciencia, la ética y el arte de enfermería a partir del conocimiento personal. Aquichán. 2009;5(1)

16. Oreyana Yañez A, Paravic Klijn T. Enfermería basada en evidencia. barreras y estrategias para su implementación. Cienc Y Enferm [Internet]. 2007;8(1):17-24. Disponible en: https://scielo.conicyt.cl/pdf/cienf/v13n1/art03.pdf

17. Queirós PJP. The knowledge in nursing and the source of this knowledge. Esc Anna Nery - Rev Enferm [Internet]. 2016;20(3):1-2. Disponible http://www.gnresearch.org/doi/10.5935/14148145.20160079

18. Cárdenas López H. El enfoque biopsicosocial y cultural en la formación de los profesionales de la salud en la
Universidad El Bosque. Procedencias, despliegues y desafíos. 1a edición. Bogotá: Universidad El Bosque; 2016. $362 \mathrm{p}$.

19. Meleis A. Theoretical Nursing: Development and Progress. 5 edición. China: Wolters Kluwer - Wolters Kluwer;

20. Rosa M, Samper M. Gestión del cuidado en Enfermería Nursing care management. Rev Cubana Enferm [Internet]. 2010;26(1):1-2. Disponible en: http://scielo.sld.cu

21. Iglesias MJS. Teoría y método: una introducción a la naturaleza del cuidado. Cult los Cuid. 2004;8(15):43-8.

22. Marie CF. Promover la vida. 2 edición. Madrid: McGrawHill; 2009.

23. Molina Restrepo M. Aprender a cuidar cuidando. Investig y Educ en Enfermería [Internet]. 2004;XXII(2):166-71. Disponible https://dialnet.unirioja.es/descarga/articulo/1321715.pdf

24. Flórez-Gutiérrez $\mathrm{C}$, Mayorga-Palacios ADP, VargasEscobar LM. La práctica formativa como escenario de aprendizaje significativo del cuidado de enfermería. Rev Colomb Enfermería [Internet]. 2017;12(14):93. Disponible en:

https://dialnet.unirioja.es/servlet/articulo? codigo $=6547210$

25. Vanegas García JH. La conciencia de la salud como expresión del cuidado. Hacia la promoción la salud [Internet]. 2017;20(2):9-10. Disponible en: http://www.scielo.org.co/pdf/hpsal/v20n2/v20n2a01.pdf

26. Boykin A, Schoenhofer S. Nursing as caring: An emerging general theory of nursing. En: Patterns of nursing theories in practice. New York: National League of Nursing; 1993. p. 83-92.

27. Boykin A, Schoenhofer S, Bulfin S, Baldwin J, McCarthy D. Living Caring in Practice: The Transformative Power of the Theory of Nursing as Caring. Int $\mathrm{J}$ Hum Caring. 2005;9(13):15-9.

28. Swanson K. Nursing as Informed Caring for the Well-Being of OthersImage. Nurs Scholarsh. 1993;25(4):352-7.

29. Algarra C, Julia A, Serrano G, Orjuela C, Marcela C, Sánchez D, et al. La filosofía de Patricia Benner y la práctica clínica Review of Patricia Benner's philosophy in clinical practice. Enfermería Glob [Internet]. 2013;32:346. Disponible http://scielo.isciii.es/pdf/eg/v12n32/revisiones5.pdf

30. Rutz Porto A, Buss Thofehrn M. El empoderamiento político de los enfermeros en la práctica hospitalaria. Index de Enfermería [Internet]. 2015;24(1-2):20-4. Disponible en: http://scielo.isciii.es/scielo.php?script=sci_arttext\&pid=S11 32-12962015000100005

31. Arabi A, Rafii F, Cheraghi, MA. Ghiyasvandian S. Nurses' policy influence: A concept analysis. Iran J Nurs Midwifery Res [Internet]. 2014;19(3):315-322. Disponible en: https://www.ncbi.nlm.nih.gov/pmc/articles/PMC4061635/

32. International Council of Nurses. Nursing Now [Internet]. 2019 [citado 24 de junio de 2019]. Disponible en: https://www.icn.ch/es/que-hacemos/campanas/nursing-now

33. Linnen D, Rowley A. Encouraging clinical nurse empowerment. Nurs Manage. 2014;45(2):44-7.

34. Manojlovich M. Power and Empowerment in Nursing: Looking Backward to Inform the Future. OJIN Online J Issues Nursing [Internet]. 22(1). Disponible en: http://ojin.nursingworld.org/mainmenucategories/anamarke tplace/anaperiodicals/ojin/tableofcontents/volume122007/n oljan07/lookingbackwardtoinformthefuture.asp

35. Duran de Villalobos MM. El cuidado: pilar fundamental de enfermería. Av en Enfermería [Internet]. 1994;12(1):16-23. Disponible 
https://revistas.unal.edu.co/index.php/avenferm/article/view $/ 16549 / 17462$

36. Reyes Gómez E. Dimensión teórico-conceptual de la enfermería disciplinar. En: Fundamentos de Enfermería Ciencia, metodología y tecnología. 2 edición. México D.F.: El manual moderno; 2015.

37. Duran de Villalobos MM. Marco epistemológico de enfermería. Aquichán [Internet]. 2002;2(2):6-18. Disponible en: http://www.scielo.org.co/pdf/aqui/v2n1/v2n1a03.pdf

38. Duran de Villalobos MM. La teoría, soporte de la ciencia y práctica de enfermería: tendencias. Av en Enfermería [Internet]. 2012;30(1):9-12. Disponible en: https://revistas.unal.edu.co/index.php/avenferm/article/view /35428/36121

39. Fink LD. Creating significant learning experiences: An integrated approach todes igning college courses. San Francisco, CA: Jossey-Bass; 2003.

40. Letelier Valdivia Mitzi VMAL. Profesionalismo en enfermería, el hábito de la excelencia del cuidado. Av en Enfermería [Internet]. 2010;28(2):145-58. Disponible en: http://www.scielo.org.co/pdf/aven/v28n2/v28n2a13.pdf

41. Duran de Villalobos MM. Concepciones de la formación del enfermero. En: Educación de enfermería en América Latina Facultad de Enfermería, Universidad Nacional de Colombia, Red de Enfermería de América Latina. Bogotá; 2000. p. 8191. 